

\title{
Computational Analysis of the Rotating Cylinder Embedment onto Flat Plate
}

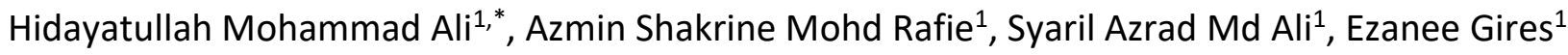 \\ 1 Department of Aerospace Engineering, Faculty of Engineering, Universiti Putra Malaysia, 43400 Serdang, Selangor, Malaysia
}

\section{ARTICLE INFO ABSTRACT}

\section{Article history:}

Received 3 October 2021

Received in revised form 15 November 2021

Accepted 1 December 2021

Available online 15 December 2021

Keywords:

Computational fluid dynamic; flat plate; Magnus effect; momentum injection; rotating cylinder

\begin{abstract}
The Magnus effect and its evolution have greatly affected the aerospace industry over the past century to date. Nevertheless, cylinder embedment onto a flat plate offers a new discovery that is yet to be investigated, specifically whether the concept could enhance the aerodynamic properties of the flat plate following the Magnus effect momentum injection. Over the past decade, the use of a rotating cylinder on an aerofoil has existed from past researches studies where the embedment has significantly increased in its aerodynamic performance better than the one without Magnus application. However, it would be hard to achieve experimental-wise as an accurate measurement and fabrication would be needed to have the same resulting effects. Here, most of the researchers would not focus deeply on the placement of the cylinder as this may increase their fabrication and testing complications. Therefore, the current study delineates the use of flat plate as the foundation design to encounter the arise matter by reducing its complication yet easy to manufacture experimentally. In this work, the model output was evaluated by using ANSYS WORKBENCH 2019 software to simulate two-dimensional flow analysis for the rotational velocities of 500 RPM and 1000 RPM, respectively. This was done for different Reynolds numbers ranging from $4.56 \mathrm{E}+05$ to $2.74 \mathrm{E}+06$ which implicitly implied with free stream velocities varying from $5 \mathrm{~m} / \mathrm{s}$ to $30 \mathrm{~m} / \mathrm{s}$ for different angles of attack between 0 to 20 degrees. Prior to developing the best model embedment, the mesh independency test was validated with an error of less than $1 \%$. The study resulted in a remarkable $C_{L}$ trend that was noticeably up to 32\% (500 RPM) and 76\% (1000 RPM) better in $C_{L}$ compared to the one without momentum injection. Similarly, the high $C_{L}$ recovery led to a tremendously lower $C_{D}$ of 51\% (500 RPM) and 99\% (1000 RPM), respectively. In sum, these findings generated a stall angle delay of up to 26\% (500 RPM) and 78\% (1000 RPM) accordingly.
\end{abstract}

\section{Introduction}

The theoretical conceptualism behind the Magnus effect is rooted in the idea that a rotating sphere or cylinder is able to generate lift in a certain direction depending on the direction of rotation. The application has been studied for almost a century to date, specifically pertaining to the embedment of rotating cylinder onto the leading edge of an aerofoil for the purpose of Magnus effect

\footnotetext{
* Corresponding author.

E-mail address: hidayatmaddali@gmail.com (Hidayatullah Mohammad Ali)
} 
implementation as lift generation and stall angle delay. However, only a few research efforts have focused on such application onto a flat plate. The flat plate is conceivably low in performance along with chain problems arising with elements such as the angle of attack $(\alpha)$, speed range, turn capability, fuel consumption, and/or any other significant characteristics. However, these issues can be countered by using a rotating cylinder to increase its aerodynamic performance. A flat plate without any boundary layer assistance, in contrast, would result in an increase in drag without any benefits for its performance prior to any plate thickness changes. It should be noted that the aerodynamic performance of a flat plate is considerably similar to that of a regular aerofoil, in addition to the existing stall.

The application of a cylinder onto bluff geometry existed back in 1925 [1]. Studies pertaining to Cylinder embedment onto Flat Plate (CyFlaP) are typically inspired by a past work known as Leading Edge Cylinder Aerofoil (LECA). In particular, Wolf and Koning [1-2] have tested the idea of LECA, which resulted in tremendous effects on the aerodynamic coefficients. Meanwhile, a study by Ahmed et al., [3] on NACA 0024 has resulted in a lift coefficient $\left(C_{L}\right)$ improvement of $36 \%$ compared to its unmodified model. Alternatively, Huda et al., [4] have investigated LECA application onto NACA 0010 and generated the maximum model lift of $145 \%$, henceforth delaying the flow separation at an increased rotation of the leading edge cylinder. Antecedent to this, Ali et al., [5] has numerically tested the embedment of rotating cylinder onto Selig S1223 aerofoil which resulted in an increase in its $C_{L}$ and stall angle delay of about $23 \%$ and $61 \%$ respectively. The $C_{L}$ is proved to rise with a rotational increment from a single rotating cylinder yet very few had been done utilizing a double rotating cylinder which may further elevate its aerodynamic performance hence providing better stability control on the aftward flow separation bubble.

Furthermore, studies on CyFlaP have only come into the picture over the last decade. In 1997, Modi [6] tested the CyFlaP configuration but merely focused on drag reduction rather than the lift increment. The study was carried out with independently rotating cylinders or those rotating in opposition for aiding the boundary layer with effective injection. Further details of the configurations and results have thus been discussed in detail in earlier publications [7-9]. Accordingly, Modi [6] also stated that the different placements of a rotating cylinder on a bluff body would change how the flow acted.

In bringing back the glory of the Magnus effect, one must know their effects on the bluff body embedment before commencing further testing, experimentally. Dozens of the references showed better improvement on their model's aerodynamic performances, yet little to none shows how was it shaped to cater to the need on achieving their embedment results. Following this, the use of a double rotating cylinder on the model would be hard to achieve on an aerofoil rather than on a flat plate. The most important aspect of achieving the cylinder to aerofoil embedment is to have an accurate and smooth surface finishing fabricated on hand, but the details are a matter of scratching one's head. However, the use of a flat plate may solve the matter and thus ease the process of achieving the results due to its straightforward design and ease to fabricate. Here, the findings of this study will redound to the benefit of researchers interested in Magnus effect flight. This work can be used as a benchmark for the future designing of an air floating platform or more. The results may be an additional point of interest to the CFD community for any numerical application of the Magnus effect in categorizing the class of unsteady flow. Therefore, this paper delineates a CyFlaP designed with a flat plate based on the patent designed by past researchers. Here, a two-dimensional (2D) flow analysis is incorporated by implementing the Computational Fluid Dynamic (CFD) method and using the Fluent analysis system from ANSYS WORKBENCH 2019 software. To this end, the embedment of CyFlaP onto the flat plate is employed to study the model efficiency for aerodynamic generation purposes. 


\section{Methodology}

The methodology used for this research was developed and described as per the following subsections and clauses. This offers an insight into the methods used for this research.

\subsection{Geometry and Computational Setup}

This study considered the proposed geometry of the cylinder and flat plate, which were set based on past research data and thus implemented onto the CFD software. The design parameter was set to account for the available resource data as follows.

\subsubsection{Cylinder}

Over the past decade, Badalamenti et al., [10] have conducted rotating cylinder testing by using a wind tunnel in the Handley Page Laboratory at City University. For the resulting rotating cylinder effects, the aerodynamic characteristics were demonstrated accordingly and then applied in comparison with other end configurations from previous research works. Furthermore, the data obtained was validated, showing an agreement with the analysis carried out by Betz [11] on the aspect ratio (AR) of 4.7. The formula used for the aspect ratio is as follows:

$$
A R=\frac{b}{c}=\frac{b}{c} * \frac{b}{b}=\frac{b^{2}}{A}
$$

where $b$ is the span, $c$ is the chord, and $A$ is the area of the model. Accordingly, the input was integrated into this work upon CFD commencement throughout the entire study.

Based on AR = 5.1 as utilized by Badalamenti et al., [10], the cylinder dimension was recorded. Therefore, the schematic diagram for a revolving cylinder with $A R=5.1$ with a diameter (D) of 0.16 $\mathrm{m}$ is shown in Figure 1 above; it rotates in a clockwise direction across an incompressible and unsteady airflow to mimic the analogous state of the experiment as performed by Badalamenti et al., [10]. Meanwhile, the clockwise rotation of the rotating cylinder was based on the analysis by Barati et al., [12] whereby the induced flow aided the free stream flow over the top of the cylinder while the flow at the bottom of the cylinder was opposed. Therefore, this condition ensured an upward lift force was generated.

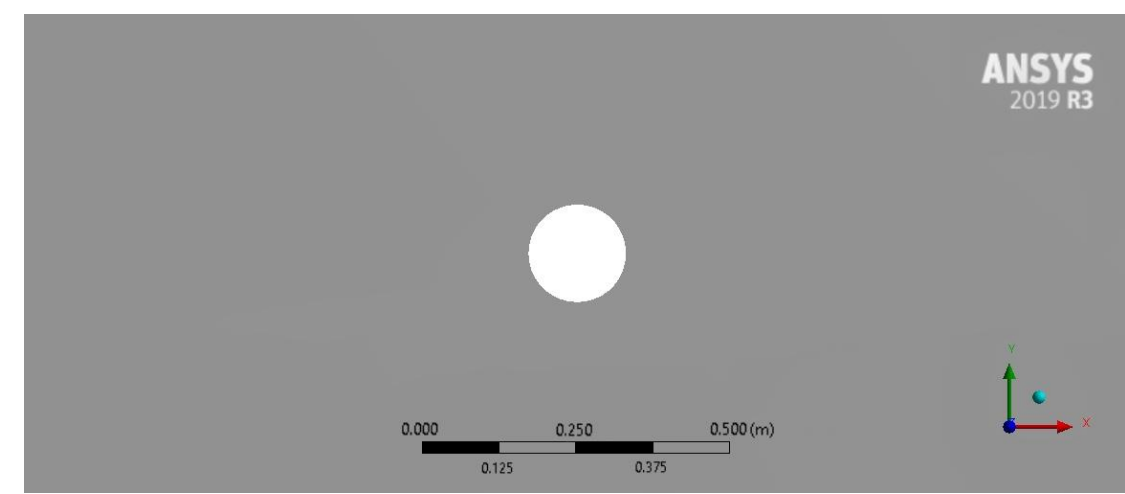

Fig. 1. The geometry of the cylinder 


\subsubsection{Flat plate}

The flat plate design was adopted as per the study by Wang et al., [13], and Mueller et al., [14] which noted the simplicity and yet lucid physical form of its design; it played a vital role as a foundation of the lift in flight history. Furthermore, the scholars revealed that the flight of a flat plate could go for a high $\alpha$ at approximately $60 \%$ of the maximum lift coefficient $\left(C_{L \max }\right)$. Therefore, the flat plate is designed with a flat plate length of $1.00 \mathrm{~m}$ as shown in Figure 2.

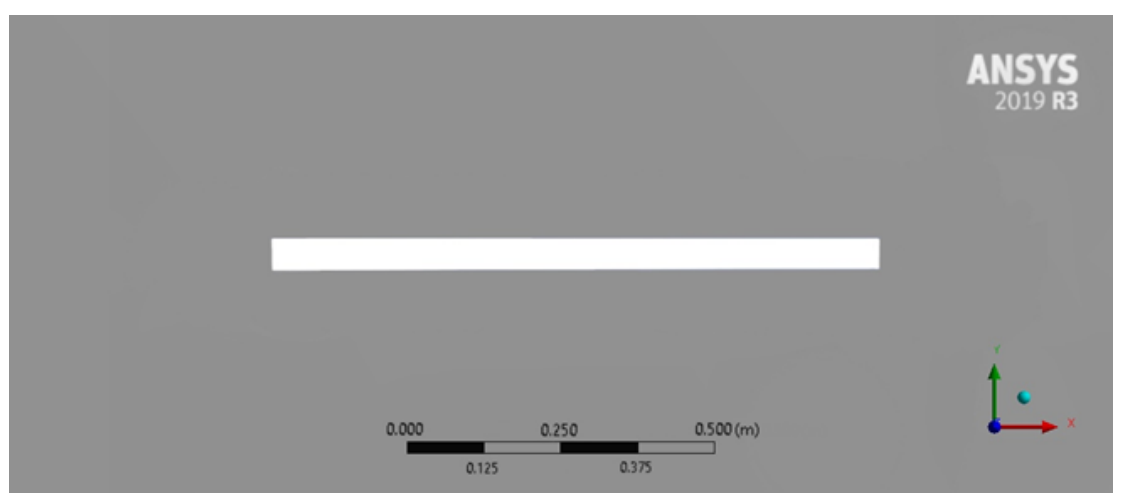

Fig. 2. The geometry of the flat plate

\subsubsection{CyFlaP}

CyFlaP was first introduced in 1997, whereby Modi ran the design aimed to reduce the drag for a truck. However, the researcher did not emphasize its lift generation, following which they noted reduced drag due to such embedment by having dual cylinder rotation on the model. Therefore, the CyFlaP design was depicted using a rotating cylinder at the leading and trailing edges both onto the flat plate, which was embedded along its camber line, and a gap of $0.005 \mathrm{~m}$ apart was ensured between the cylinder and embedded flat plate as shown in Figure 3. The gap was applied prior to the study by Abdulla et al., [15], which established an ideal configuration for the performance of the CyFlaP.



Fig. 3. The geometry of the CyFlaP

\subsection{Turbulence Model}

By definition, turbulence modeling denotes a computational procedure geared for closing a system of mean flow equations. It describes the design and usage of mathematical models to solve or forecast the impacts of turbulence, whereby its governing equations of turbulence often offer 
simplified solutions by using the averaging method. For the current work, the K- $\omega$ model was used with reference from the research by Mgaidi et al., [16] as a two-equation model, it incorporated two extra transport equations to delineate the turbulent flow properties accordingly. Here, the turbulent kinetic energy is denoted as $\mathrm{K}$ as the first transported variable, while the specific dissipation is denoted as $\omega$ to indicate the second transported variable.

\subsubsection{Shear stress transport $K-\omega$ model}

In 1994, Menter [17] developed a two-equation eddy-viscosity model known as the Shear Stress Transport (SST) K-w turbulence model, which was utilized to blend the $\mathrm{K}-\omega$ model in the near-wall region with the free stream $\mathrm{K}-\omega$ model in the far-field. This was an improvement to the initial model presented by Wilcox [18], which was capable of depicting good turbulence formulation for the adverse pressure gradients and separating flow both. In contrast, the SST formulation was formed from modeling $\mathrm{K}-\omega$ and $\mathrm{K}-\varepsilon$ both together, thereby aiding the switch from the former to the latter in preventing problems arising in the inlet of the free-stream turbulence properties. Concurrently, the $\mathrm{K}-\omega$ formulation was implemented at the inner part of the boundary layer instead of the $\mathrm{K}-\varepsilon$ formulation. In Fluent, the general form of the SST K- $\omega$ transport equations are shown as follows:

$$
\begin{aligned}
& \frac{\partial}{\partial t}(\rho k)+\frac{\partial}{\partial x_{i}}\left(\rho k u_{i}\right)=\frac{\partial}{\partial x_{j}}\left[\Gamma_{k} \frac{\partial k}{\partial x_{j}}\right]+G_{k}-Y_{k}+S_{k} \\
& \frac{\partial}{\partial t}(\rho \omega)+\frac{\partial}{\partial x_{i}}\left(\rho \omega u_{i}\right)=\frac{\partial}{\partial x_{j}}\left[\Gamma_{\omega} \frac{\partial \omega}{\partial x_{j}}\right]+G_{\omega}-Y_{\omega}+S_{\omega}
\end{aligned}
$$

In these equations, the generation of turbulence kinetic energy attributable to mean velocity gradient and production of $\omega$ is represented as $G_{k}$ and $G_{\omega}$, respectively. Meanwhile, the effective diffusivity of $k$ and $\omega$ due to turbulence is represented as $\Gamma_{\mathrm{k}}$ and $\Gamma_{\omega}$, respectively. Then, the dissipation of $k$ and $\omega$ is represented as $Y_{k}$ and $Y_{\omega}$, respectively, while $D_{\omega}$ represents the crossdiffusion term. Last but not least, the $S_{k}$ and $S_{\omega}$ are user-defined source terms.

Regarding the aforementioned statement, the SST K- $\omega$ model shows similarity with the standard $\mathrm{K}-\omega$ model; however, it is inclusive of the following refinements [19]:

a. A blending function is used to multiply the standard $\mathrm{K}-\omega$ model with the transformed $\mathrm{K}-$ $\omega$ model, which are then added together.

b. In the blending function, the standard $\mathrm{K}-\omega$ model will be activated for a near-wall condition, while the transformed $\mathrm{K}-\omega$ model will be activated zero away from the surface.

c. The $\omega$ equation is derived as a dampened cross-diffusion in the SST model.

d. The difference between the turbulent shear stress transport and the modeling constant is accounted for by modifying the turbulent viscosity definition.

All and all, such refinements would render the K- $\omega$ SST model highly accurate and reliable in comparison with the standard K- $\omega$ model for a broader range of flows, inclusive aerofoils, adverse pressure gradient flows, and transonic shock waves [20]. 


\subsection{Key Performance Parameter}

In this analysis, the Key Performance Parameters (KPPs) explicitly and specifically define the output target. Here, the Reynolds number $(R e)$ is one of the major elements in predicting the flow patterns across different fluid conditions, which is defined as follows:

$$
\operatorname{Re}=\frac{\rho * v * \ell}{\mu}
$$

where $\rho$ is the fluid density (i.e., air), $V$ is the inlet velocity, $\ell$ represents the model length or diameter, and $\mu$ represents the dynamic viscosity of the fluid. The Re used for this study is shown in Table 1 according to their inlet velocity.

Table 1

Reynold number based per inlet velocity

\begin{tabular}{ll}
\hline Reynold number & Inlet Velocity \\
\hline $4.56 \times 10^{5}$ & $5 \mathrm{~m} / \mathrm{s}$ \\
$9.12 \times 10^{5}$ & $10 \mathrm{~m} / \mathrm{s}$ \\
$1.37 \times 10^{6}$ & $15 \mathrm{~m} / \mathrm{s}$ \\
$1.82 \times 10^{6}$ & $20 \mathrm{~m} / \mathrm{s}$ \\
$2.28 \times 10^{6}$ & $25 \mathrm{~m} / \mathrm{s}$ \\
$2.74 \times 10^{6}$ & $30 \mathrm{~m} / \mathrm{s}$ \\
\hline
\end{tabular}

Eq. (5) and Eq. (6) below are the lift coefficient $\left(C_{L}\right)$ and drag coefficient $\left(C_{D}\right)$ equations used for model formulation. The equations are as follows:

$$
\begin{gathered}
C_{L}=\frac{L}{\frac{1}{2} * \rho * \mathrm{~V}^{2} * S} \\
C_{D}=\frac{D}{\frac{1}{2} * \rho * V^{2} * S}
\end{gathered}
$$

where $L$ is the lift force, $D$ is the drag force, and $S$ is the projected area of the model.

\subsection{Grid Generation}

The standard CFD method would require a mesh suited for the boundaries of the computational domain. This necessitates the generation of a computational mesh befitting the intended 2D NavierStokes equations, wherein the domain and its boundaries are thus specified with a set of grid points. Such a grid system is known as grid generation.

\subsubsection{Mesh topology}

In the Fluent analysis system, two meshing zones were incorporated and consisted of an inner zone with a 2D $\times 3 \mathrm{D}$ block across the model and an outer zone with a $6 \mathrm{D} \times 15 \mathrm{D}$ block setup surrounding the inner zone (refer Figure 4(a)). The block setup was implemented according to the research works by Mgaidi et al., [16], and Yao et al., [21]. Moreover, the use of a longer aftward 
domain may reduce the probability of getting reverse wind flow into the domain from the outlet. The model surfaces were then fixed with $y$-plus $(y+)$ less than 1 , which was positioned from the wall to the first mesh node by a maximum of 15 layers of inflation requirement and together with a growth rate of 1.2 to achieve good results (see Figure $4(\mathrm{~b})$ and $4(\mathrm{c})$ ). Here, the skewness and orthogonal quality of the meshing had surpassed the metrics spectrum of less than 0.8 and more than 0.2 respectively.

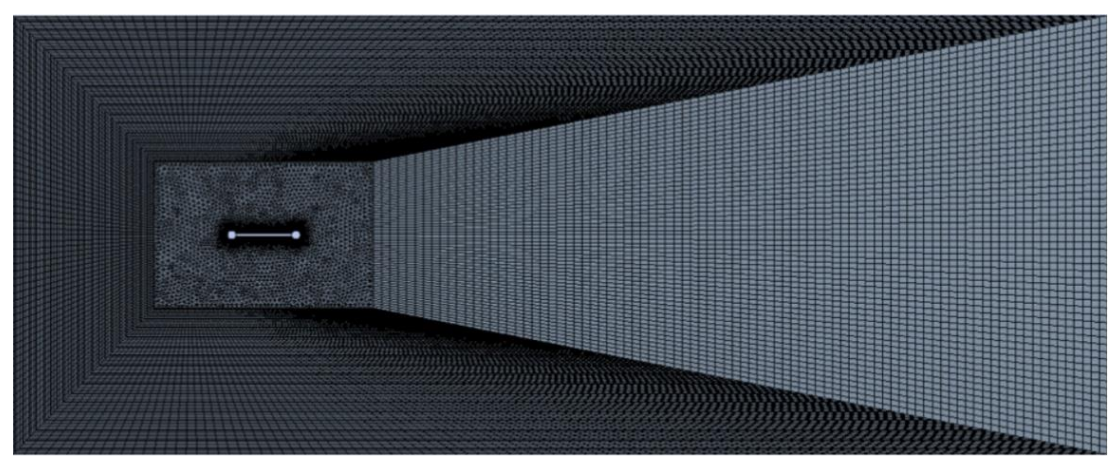

(a)

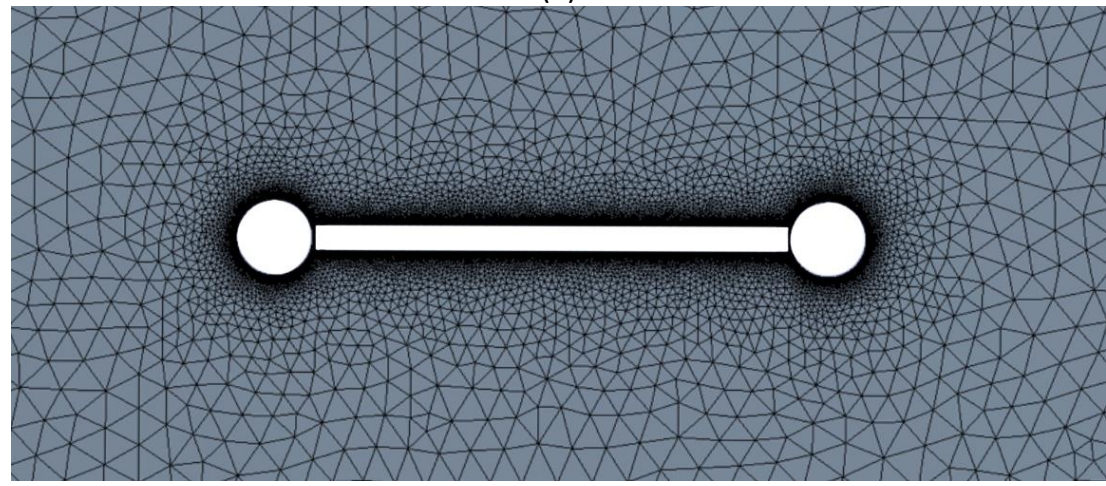

(b)

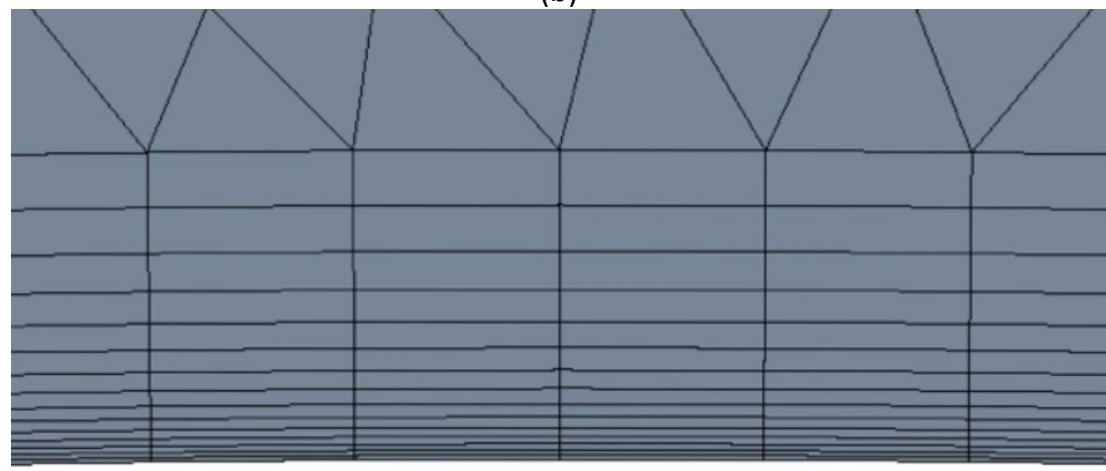

(c)

Fig. 4. Domain and mesh generation for the CyFlaP model (a) domain configuration (b) mesh applicated on the model's wall $(c)$ the inflation from the first layer thickness

Furthermore, the wall function strategy could be comfortably accomplished for a specific turbulence model. As per Tables 2 and 3, the specifications of the boundary conditions and mesh environment for this analysis are given. 
Table 2

Specifications of boundary conditions

\begin{tabular}{ll}
\hline Boundary conditions & Type \\
\hline Inlet & Velocity-inlet \\
Outlet & Pressure-outlet \\
Cylinder & Wall \\
Selig aerofoil & Wall \\
Wall & Symmetry \\
Interior surface body & Interior \\
Surface body & Interior \\
\hline
\end{tabular}

\section{Table 3}

Mesh configurations

\begin{tabular}{ll}
\hline Mesh specifications & \\
\hline Growth rate & 1.2 \\
Defeature size & $5 . e-004 \mathrm{~m}$ \\
Curvature minimum size & $1 . \mathrm{e}-003 \mathrm{~m}$ \\
Curvature normal angle & $18.0^{\circ}$ \\
Smoothing & High \\
\hline Inflation specifications & \\
\hline Inflation option & First layer thickness \\
Maximum layers & 15 \\
Growth rate & 1.2 \\
\hline
\end{tabular}

\subsubsection{Mesh independency test}

A mesh independence test (MIT) was recommended to monitor the solution grid independence before proceeding with the model testing phase. This was achieved by constructing a new grid of cells and evaluating it using several acquired alternatives. Therefore, MIT is carried out for the rotating cylinder and flat plate both as seen in Figures 5(a) and 5(b).

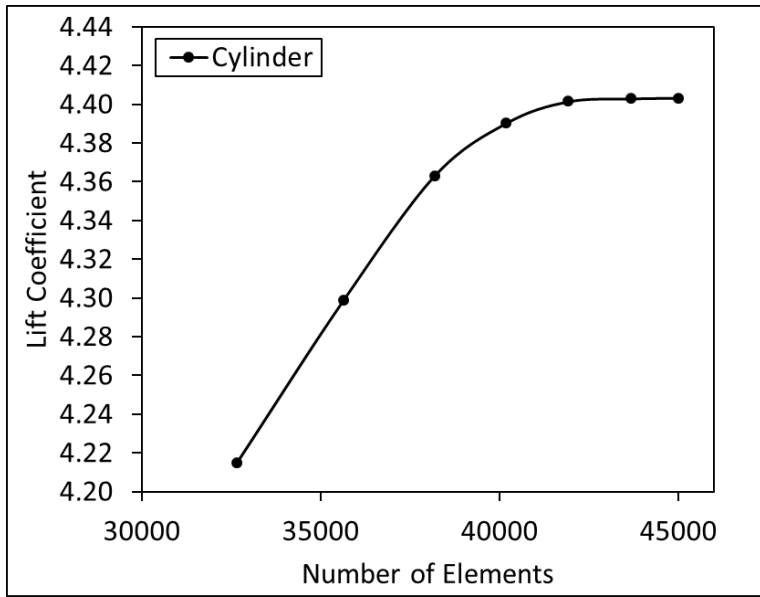

(a)

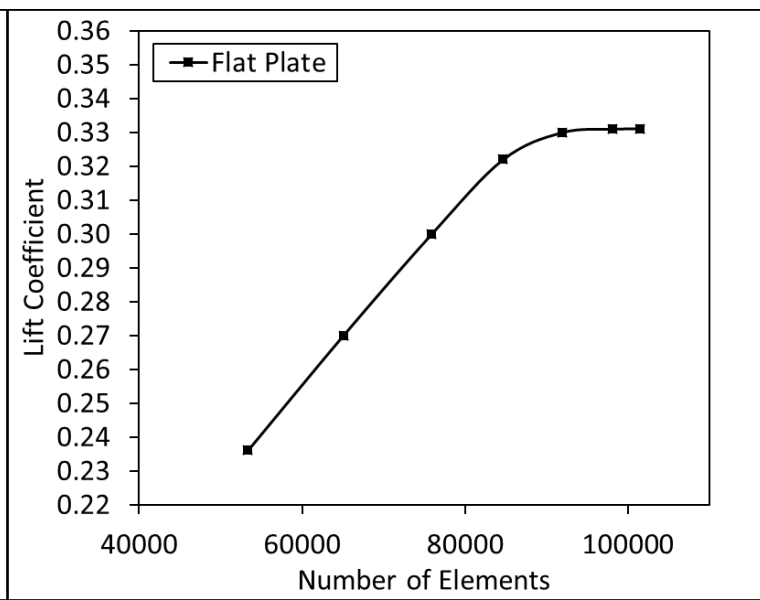

(b)

Fig. 5. MIT for the rotating cylinder (a) and flat plate (b)

Before obtaining the highest data quality, two MITs were conducted. The grid refinement for $C_{L}$ at approximately $4.19 \mathrm{E}+04$ (rotating cylinder) and 9.19E+04 (flat plate) cells resulted in the highest precision and durability for CFD research from now on. To attain the optimal time efficiency, the percentage of error for the above-mentioned grid refinement was less than $1 \%$. It is important to test 
out the MIT prior hand before commencing further into the computational testing to ensue in a suitable and best mesh refinement for any models.

\subsection{Solver Setting}

The appropriate setting for the intended condition must be set in the CFD solver interface before it is running for calculation solutions. Table 4 shows the best setup implemented in ascertaining the numerical simulations. Here, the table has been sectioned into five parts consisting of general setting, viscous model, reference values, solution setting, and partial discretization. A standard practice of setting the residual error to 1.e-006 was used to lessen the resulting error.

Table 4

ANSYS Fluent 19 solver setting

\begin{tabular}{ll}
\hline General & \\
\hline Type & $\begin{array}{l}\text { Pressure-based } \\
\text { Aelocity formulation }\end{array}$ \\
$\begin{array}{l}\text { Trme } \\
\text { Transient } \\
\text { Planar }\end{array}$ \\
\hline Model & \\
\hline Viscous & SST k-omega \\
\hline Reference values & \\
\hline Density (kg/m3) & 1.225 \\
Pressure (pascal) & 101325 \\
Temperature (K) & 288.16 \\
Velocity (m/s) & $5,10,15,20,25,30$ \\
Viscosity (kg/m-s) & $1.789 e-005$ \\
\hline Solution & \\
\hline Method & Pressure-velocity coupling \\
Scheme & Coupled \\
Residual error & $1 . e-006$ \\
\hline Spatial discretization & \\
\hline Gradient & Green Gauss Node Based \\
Pressure & PRESTO! \\
Momentum & QUICK \\
Turbulent kinetic energy & QUICK \\
Specific dissipation rate & QUICK \\
\hline
\end{tabular}

\subsection{Validation}

Before commencing further into the CFD process, validation has been studied for both the cylinder and flat plate as to achieve finer results (refer to Figures 6(a) and 6(b)). The embedment of the cylinder and the flat plate was carefully done based on this validation study. Badalamenti et al., [10], and Mueller et al., [14] have undergone experimental fluid dynamic (EFD) about the rotating cylinder and flat plate which are then verified in this validation study by using the CFD approach. Furthermore, this validation would further justify the correct use of meshing and solver settings. The validation study has resulted in an error of less than $10 \%$ for both of the conditions. 


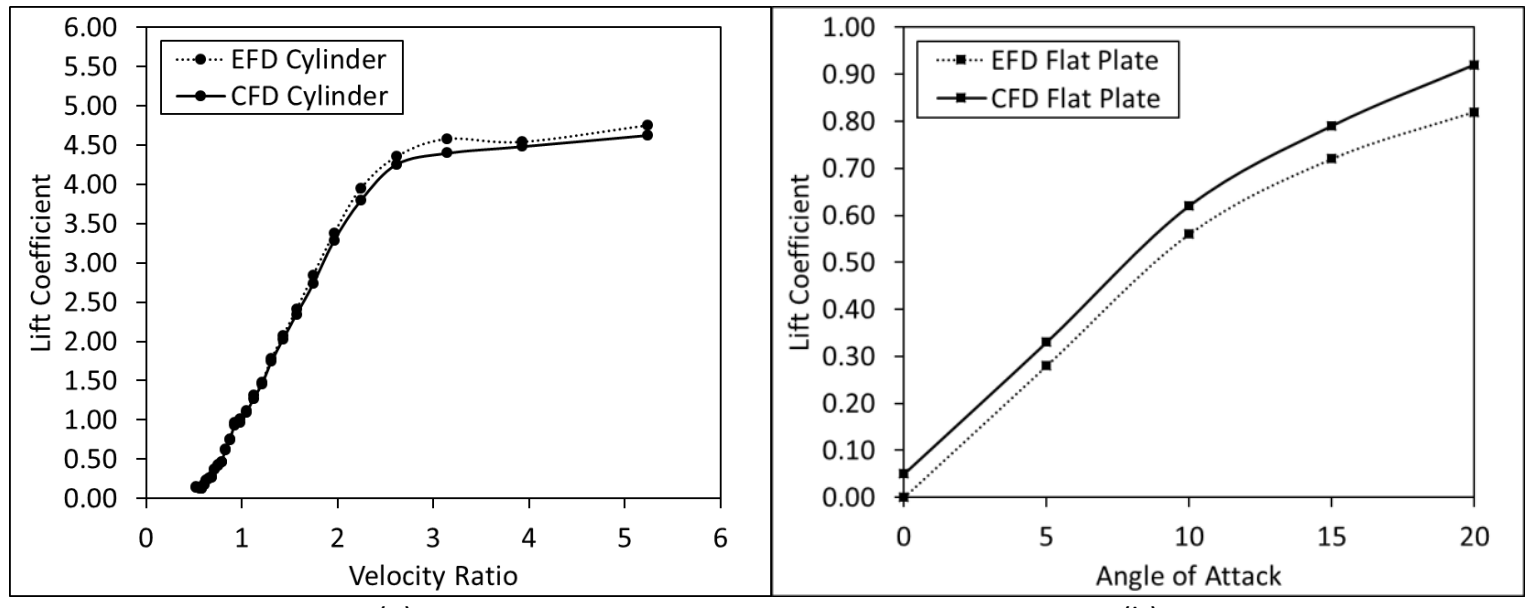

(a)

(b)

Fig. 6. Validation for the rotating cylinder (a) and flat plate (b)

\section{Results and Discussion}

The computational outcomes for the CyFlaP are computed using the following parameters: 1) Re values of $4.56 \times 10^{5}, 9.12 \times 10^{5}, 1.37 \times 10^{6}, 1.82 \times 10^{6}, 2.28 \times 10^{6}$, and $2.74 \times 10^{6} ; 2$ ) with a cylinder rotational speed in revolution per minute (RPM) of 0 RPM, 500 RPM, and 1000 RPM; and 3 ) at 5 degrees $\left({ }^{\circ}\right.$ ) interval of $\alpha$ from $0^{\circ}$ to $20^{\circ}$. Consequently, the resultant forces on CyFlaP yielded two forces, whereby the net force component acting normal to the velocity inlet is known as the lift force while the net force component acting parallel to the velocity inlet is known as the drag force.

\subsection{Numerical Simulation}

The following clauses are the computational results about the aerodynamic data as $C_{L}, C_{D}$, $R P M, \operatorname{Re}$, and $\alpha$, which yield a relationship in the output of the computational results.

\subsubsection{Effect of magnus effect}

The computational study for the proposed CyFlaP results in the following outcomes as shown in Figures 7 to 9 . These figures display the effect of the Magnus effect on the CyFlaP by having a momentum injection of 0 RPM, 500 RPM, and 1000 RPM, respectively.

The $C_{L}$ of the CyFlaP in Figure 7(a) denotes a decrease along with the increasing Re for the 0 RPM momentum injection condition. This was attributable to the earlier formation of turbulent flow and boundary layer separation that occurred, causing a decrement in the lift, an increase in drag, and reduced in speed thus only showing a high lift character at low Re at the moment. Basu [22], and Hakim et al., [23] denoted that the significant reduction in the velocity of the boundary layer is due to the adverse pressure gradient that occurred when the static pressure aftward increases with the direction of the flow. This matter resulted in a flow reversal because the kinetic energy of the fluid particle is no longer adequate to move the particle against the pressure gradient. Therefore, the boundary layer had a partial detachment from the surface body [24]. 




(a)

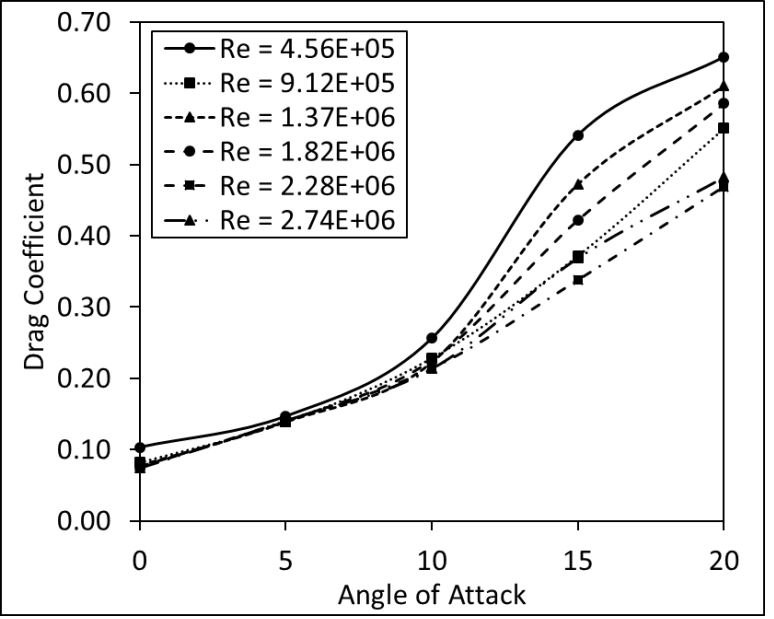

(b)

Fig. 7. $C_{L}(\mathrm{a})$ and $C_{D}$ (b) of the CyFlaP versus $\alpha$ and Re at 0 RPM of rotational speed

However, the $C_{L}$ increased with an increment of the $\alpha$, as well as having a rising trend of $C_{L}$ that was noticeably up to $32 \%$ (500 RPM) and 76\% (1000 RPM) better in $C_{L}$ compared to its unmodified model without any momentum injection. The high $C_{L}$ recovery resulted in a tremendously lower $C_{D}$ of 51\% (500 RPM) and 99\% (1000 RPM), respectively, thereby yielding a stall angle delay of up to $26 \%$ (500 RPM) and 78\% (1000 RPM).

Nevertheless, the $C_{L}$ for both 500 RPM and 1000 RPM yields better performance at $0^{\circ}$ (refer to Figures 8(a) and 9(a)). The resulted high in $C_{L}$ hereby be beneficial of the lift generation for the CyFlaP without requiring a high power usage as a start-up. A noticeable plateau effect is noticed as shown in Figures 8(a) and 9(a), whereby the rotating speed of 500 RPM and 1000 RPM ranging from $5^{\circ}$ to $15^{\circ}$ is seen for the high Re condition.

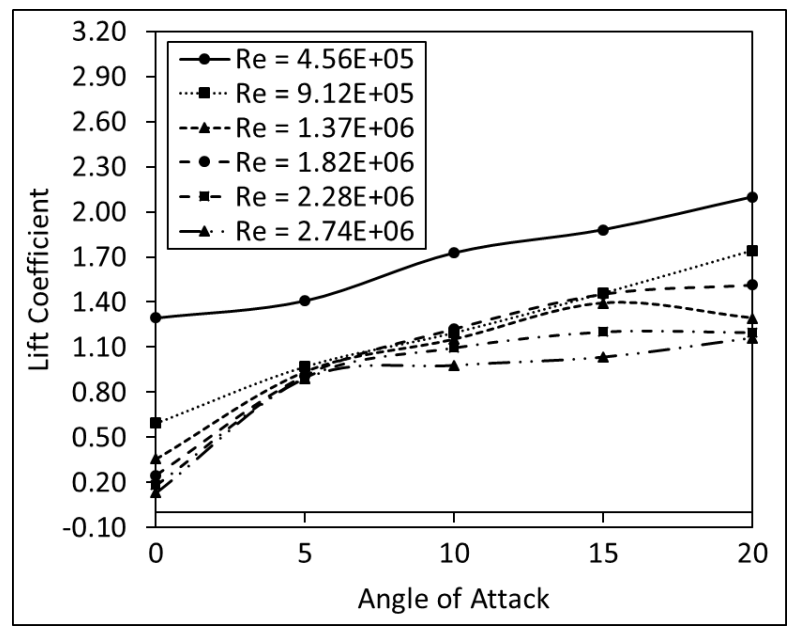

(a)



(b)

Fig. 8. $C_{L}$ (a) and $C_{D}$ (b) of the CyFlaP versus $\alpha$ and Re at 500 RPM of rotational speed 


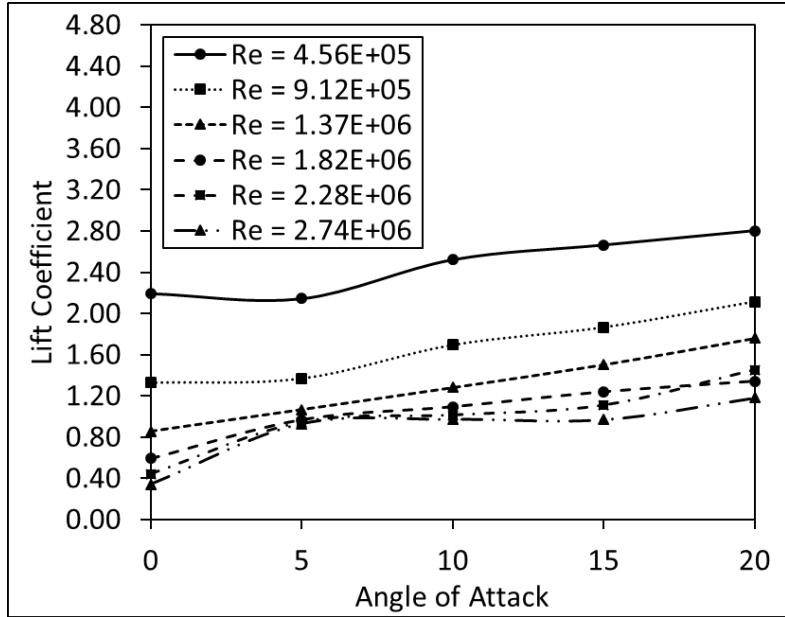

(a)

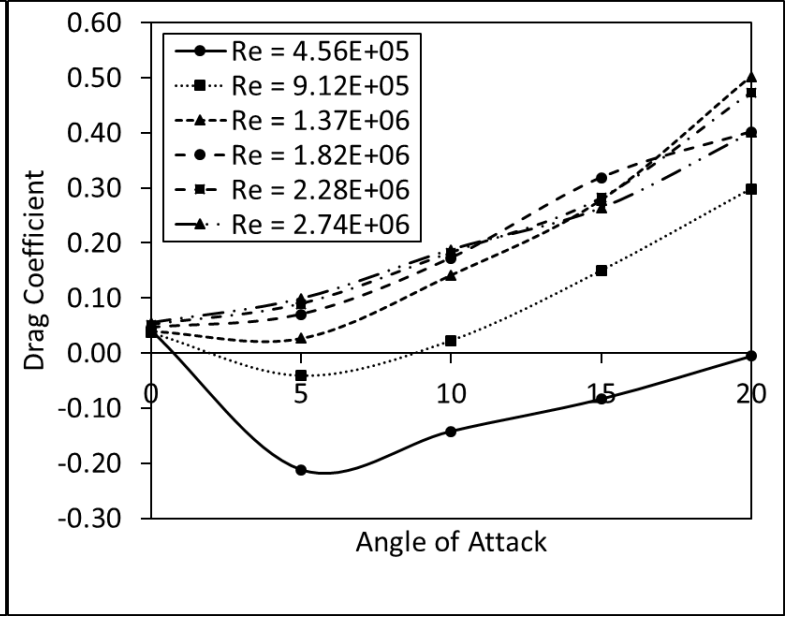

(b)

Fig. 9. $C_{L}(\mathrm{a})$ and $C_{D}$ (b) of the CyFlaP versus $\alpha$ and Re 1000 RPM of rotational speed

The sudden plateau effect was attributed to partial flow separation and contributed to the existing separation bubble. Hence, an increment in momentum injection would enhance and thus reduce the effect, namely by reattaching the air stream towards the surface body to ensure a steady separation control throughout the elevated $\alpha$. Besides, a higher momentum injection led to a steady increment of $C_{L}$ yet lower in $C_{D}$, which further elevated the aerodynamic performance of the CyFlaP. This momentum injection acted as an active flow control method to increase the boundary layer energy so that it can keep on attaching to the profile yet delaying the aerofoil flow separation [25].

Therefore, with the assisted Magnus effect for momentum injection on the CyFlaP, it can be denoted that the CyFlaP behaved the best and was stable at a low Re and could achieve a superb result if it was capable of utilizing a grit roughness or modifier on the cylinder surface as per the study by Marzuki et al., [26], and Merryisha et al., [27]. Further research on varying the momentum injection would be highly beneficial in improving the aerodynamic performance of the CyFlaP.

\subsubsection{Effect on velocity magnitude contour}

The physical vector quantity for this study was denoted as velocity as per the velocity inlet of 5 $\mathrm{m} / \mathrm{s}$ to $30 \mathrm{~m} / \mathrm{s}$. The input velocity is yet to be implemented in the Re formula to predict the pattern of a situational fluid flow, whereby the direction and magnitude are necessary for defining the velocity while the speed is the scalar absolute value for the velocity magnitude. The velocity magnitude contour for eighteen (18) different conditions at $\alpha$ of $20^{\circ}$ for Re $4.56 \mathrm{E}+05$ to $2.74 \mathrm{E}+06$ at three (3) different RPM of 0, 500, and 1000 RPM are presented in Figure 10. 


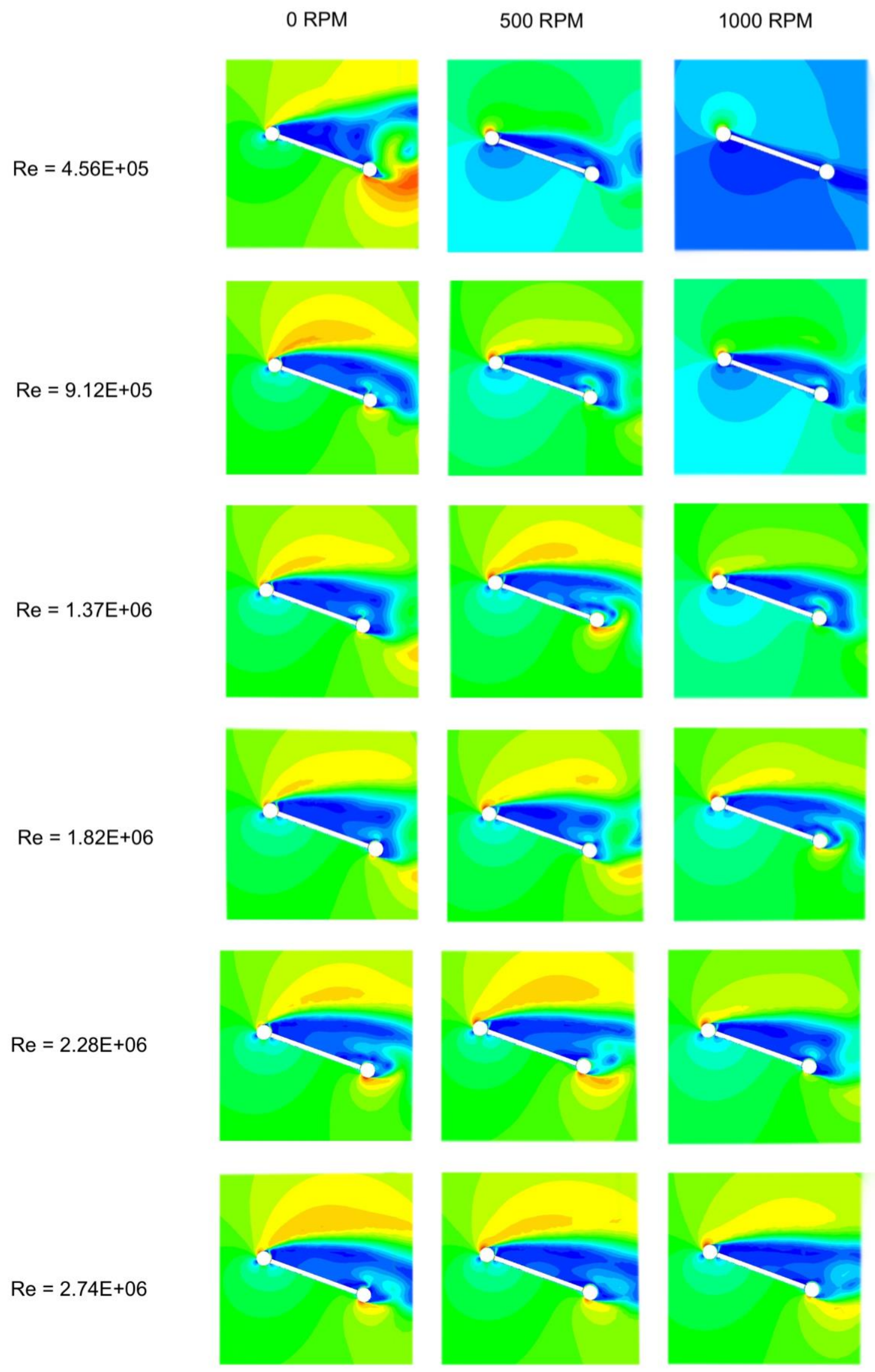

Fig. 10. Flow evolution for velocity magnitude contour 
Here, swirling vortices were found behind the CyFlaP when no rotation (0 RPM) of the rotating cylinder was applied. Meanwhile, the velocity magnitude contour with the momentum injection of 500 RPM and 1000 RPM depicted that the contour on the upper surface of CyFlaP was elongated and reduced in vortices towards an increment of momentum injection. Above the CyFlaP, an increase in momentum injection resulted in a decrease in pressure, which would be explained in the later clauses. Based on the Bernoulli principle, the airflow on the lower pressure region would speed up by a steady airflow of constant energy. Therefore, this resulted in the contribution of steady velocity magnitude with a better boundary condition.

\subsubsection{Effect on pressure coefficient contour}

The pressure coefficient $\left(C_{P}\right)$ contour as shown in Figure 11 is extracted from the CFD postprocessing in determining its effect on the flow evolution for pressure distribution along the CyFlaP's surface body. In this study, 18 different conditions were laid out at $\alpha$ of $20^{\circ}$ for $\operatorname{Re} 4.56 \mathrm{E}+05$ to $2.74 \mathrm{E}+06$ across three different RPM values, namely 0, 500, and 1000 RPM. Here, a flow separation following the CyFlaP existed, thus indicating the existence of Kármán vortex street for the 0 RPM condition. The Kármán vortex street is a condition of swirling vortices that appear on a model, worsening the pre-existing flow over time. The high flow separation is expected to exist when no injection is applied onto the embedded flat plate, thus indicating the vital role of the momentum injection in improving the model boundary layer flow. However, the separation flow was reduced when momentum injection of 500 RPM and 1000 RPM was applied, thereby further reattaching the flow onto the CyFlaP wall, and led to an increase in its lift and a reduced drag generation.

Bernoulli's principle of having a low pressure on the upper surface and high pressure on the lower surface of the model successfully resulted in lift generation. Besides, the formation of high-pressure flow acting on the lower surface of the model that was wider in size when a momentum injection was applied was seen. Such formation of flows indicated that the lift was increased while also reducing the drag acted upon an increment of the momentum injection. This further delayed and extended the stall angle of the model. 


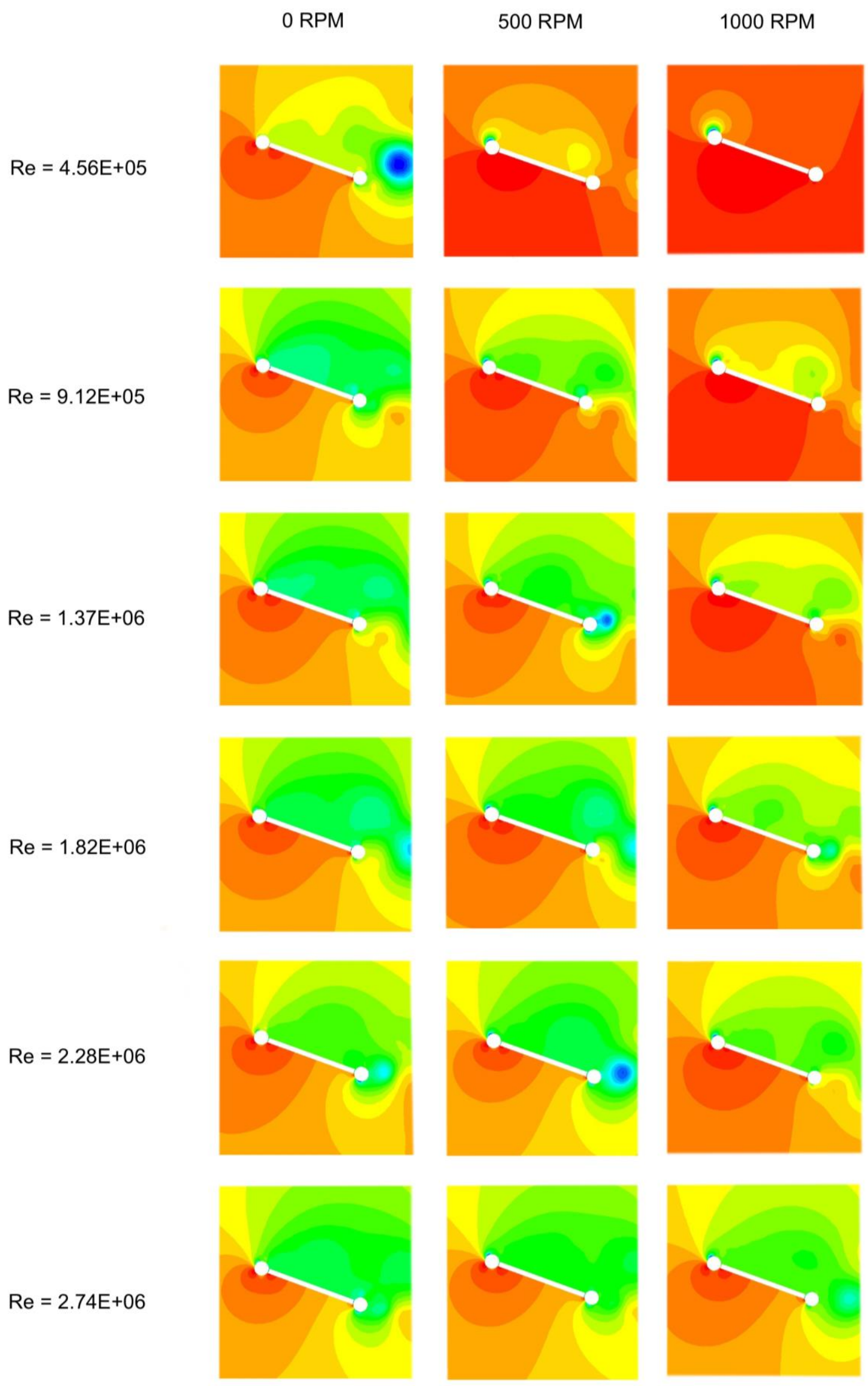

Fig. 11. Flow evolution for $C_{P}$ contour 


\section{Conclusion}

In brief, the computational analysis of a rotating cylinder embedment onto a flat plate for improving the surface boundary layer (i.e., the CyFlaP) resulted in a tremendous effect on the model's aerodynamic performance. Moreover, a superb $C_{L}$ trend was noticeable, documenting improvements up to 32\% (500 RPM) and 76\% (1000 RPM) compared to the one without momentum injection. The high $C_{L}$ recovery resulted in a tremendously lower $C_{D}$ value of 51\% (500 RPM) and $99 \%$ (1000 RPM), respectively, thereby resulting in a stall angle delay of up to $26 \%$ (500 RPM) and $78 \%$ (1000 RPM). Accordingly, it can be denoted that the CyFlaP behaved the best and was stable at a low Re and could achieve a superb result if it was capable of utilizing a grit roughness or modifier on the cylinder surface. All and all, the higher momentum injection discerned the rise in $C_{L}$, reduction in $C_{D}$, and extended stall angle delay, thus depicting a successful Magnus effect application on the model. Therefore, the CyFlaP would be beneficial for an integrated application in the aerospace world soon.

\section{Acknowledgment}

This research was funded by a grant from the Ministry of Education (MOE) Malaysia through Fundamental Research Grant Scheme (FRGS/1/2018/TK09/UPM/02/2).

\section{References}

[1] Wolff, E. B. Preliminary Investigation of the Effect of a Rotating Cylinder in a Wing. No. NACA-TM-307. 1925.

[2] Wolff, E. B., and C. Koning. Tests for determining the effect of a rotating cylinder fitted into the leading edge of an airplane wing. No. NACA-TM-354. 1926.

[3] Ahmed, S., A. Nazari, and E. Wahba. "Numerical analysis of separation control over an airfoil section." International Review of Aerospace Engineering 7, no. 2 (2014): 61-68. https://doi.org/10.15866/irease.v7i2.2057

[4] Huda, Md Nurul, Tabassum Ahmed, T. S. M. Ahmed, M. A. Salam, Md Rayhan Afsar, Kh Md Faisal, and MA Taher Ali. "Study of NACA 0010 symmetric airfoil with leading edge rotating cylinder in a subsonic wind tunnel." In 11th International Conference on Mechanical Engineering, BUET, Dhaka, Bangladesh. 2015.

[5] Ali, Hidayatullah Mohammad, Azmin Shakrine Mohd Rafie, and Syaril Azrad Md Ali. "Numerical Analysis of Leading Edge Cylinder Aerofoil on Selig S1223 for Moving Surface Boundary Control." Journal of Aeronautics, Astronautics and Aviation 53, no. 2 (2021): 143-153. https://doi.org/10.6125/JoAAA.202106 53(2).06

[6] Modi, V. J. "Moving surface boundary-layer control: A review." Journal of fluids and structures 11, no. 6 (1997): 627-663. https://doi.org/10.1006/ifls.1997.0098

[7] Modi, V. J., M. S. U. K. Fernando, and T. Yokomizo. "Moving surface boundary layer control as applied to two- and three-dimensional bodies." Proceedings of the 8th Colloquium on Industrial Aerodynamics-Industrial Flows (eds.), C Kramer, H Gerhardt (Aachen, Germany: Fachhoehschule), (1989): 73-84.

[8] Modi, V., M. S. U. K. Fernando, and T. Yokomizo. "Drag reduction of bluff bodies through moving surface boundary layercontrol." In 28th Aerospace Sciences Meeting, p. 298. 1990. https://doi.org/10.2514/6.1990-298

[9] Modi, V. J., M. S. U. K. Fernando, and T. Yokomizo. "Moving surface boundary-layer control as applied to twodimensional and three-dimensional bluff bodies." Journal of Wind Engineering and Industrial Aerodynamics 38, no. 1 (1991): 83-92. https://doi.org/10.1016/0167-6105(91)90029-V

[10] Badalamenti, Carmine, and Simon Prince. "Effects of endplates on a rotating cylinder in crossflow." In 26th AIAA Applied Aerodynamics Conference, p. 7063. 2008. https://doi.org/10.2514/6.2008-7063

[11] Betz, A. "Der magnuseffekt, die grundlage der flettner-walze." Zeitschrift des vereins deutscher Ingenieure. Translated to: The "Magnus Effect" The Principle of the Flettner rotor. NACA Technical Memorandum, TM310 (1925): 9-14.

[12] Barati, Ebrahim, Mehdi Rafati Zarkak, and Javad Abolfazli Esfahani. "Effect of Rotational Direction of Circular Cylinder for Mixed Convection at Subcritical Reynolds Number."

[13] Wang, Shizhao, Xing Zhang, Guowei He, and Tianshu Liu. "A lift formula applied to low-Reynolds-number unsteady flows." Physics of Fluids 25, no. 9 (2013): 093605. https://doi.org/10.1063/1.4821520

[14] Mueller, Thomas J., and Gabriel E. Torres. Aerodynamics of low aspect ratio wings at low Reynolds numbers with applications to micro air vehicle design and optimization. NOTRE DAME UNIV IN OFFICE OF RESEARCH, 2001. https://doi.org/10.21236/ADA397533 
[15] Abdulla, Najdat Nashat, and Mustafa Falih Hasan. "Effect of Gap between Airfoil and Embedded Rotating Cylinder on the Airfoil Aerodynamic Performance." https://doi.org/10.31031/RDMS.2018.03.000567

[16] Mgaidi, A. M., AS Mohd Rafie, K. A. Ahmad, R. Zahari, MF Abdul Hamid, and O. F. Marzuki. "NUMERICAL AND EXPERIMENTAL ANALYSES OF THE FLOW AROUND A ROTATING CIRCULAR CYLINDER AT SUBCRITICAL REGIME OF REYNOLDS NUMBER USING K-E AND K- $\Omega$-SST TURBULENT MODELS." (2006).

[17] Menter, Florian R. "Two-equation eddy-viscosity turbulence models for engineering applications." AIAA journal 32 , no. 8 (1994): 1598-1605. https://doi.org/10.2514/3.12149

[18] Wilcox, David C. "Reassessment of the scale-determining equation for advanced turbulence models." $A I A A$ journal 26, no. 11 (1988): 1299-1310. https://doi.org/10.2514/3.10041

[19] Khalil, Hesham, Khalid Saqr, Yehia Eldrainy, and Walid Abdelghaffar. "Aerodynamics of a trapped vortex combustor: A comparative assessment of RANS based CFD models." Journal of Advanced Research in Fluid Mechanics and Thermal Sciences 43, no. 1 (2018): 1-19.

[20] Khan, Sher Afghan, Musavir Bashir, Maughal Ahmed Ali Baig, and Fharukh Ahmed Ghasi Mehaboob Ali. "Comparing the Effect of Different Turbulence Models on The CFD Predictions of NACA0018 Airfoil Aerodynamics." CFD Letters 12, no. 3 (2020): 1-10. https://doi.org/10.37934/cfdl.12.3.110

[21] Yao, Q., C. Y. Zhou, and C. Wang. "Numerical Study of the Flow past a Rotating Cylinder at Supercritical Reynolds Number." In 4th International Conference on Mechanical Materials and Manufacturing Engineering (MMME 2016). 2016. https://doi.org/10.2991/mmme-16.2016.159

[22] Basu, P. "Boundary layer with pressure gradient." Greenfield Research Inc., Greenfield Research Inc. 18 (2001).

[23] Hakim, Muhammad Syahmi Abdul, Mastura Ab Wahid, Norazila Othman, Shabudin Mat, Shuhaimi Mansor, Md Nizam Dahalan, and Wan Khairuddin Wan Ali. "The effects of Reynolds number on flow separation of Naca Aerofoil." Journal of Advanced Research in Fluid Mechanics and Thermal Sciences 47, no. 1 (2018): 56-68.

[24] Hamisu, Muhammad Tukur, Mahmud Muhammad Jamil, Umar Sanusi Umar, and Aisha Sa'ad. "Numerical Study Of Flow In Asymmetric 2D Plane Diffusers With Different Inlet Channel Lengths." CFD Letters 11, no. 5 (2019): 1-21.

[25] Liang, Chua Bing, Akmal Nizam Mohammed, Azwan Sapit, Mohd Azahari Razali, Mohd Faisal Hushim, Amir Khalid, and Nurul Farhana Mohd Yusof. "Numerical Simulation of Aerofoil with Flow Injection at the Upper Surface." (2021).

[26] Marzuki, Omar Faruqi, Azmin Shakrine Mohd Rafie, Fairuz Izzuddin Romli, and Kamarul Arifin Ahmad. "Magnus wind turbine: the effect of sandpaper surface roughness on cylinder blades." Acta Mechanica 229, no. 1 (2018): 7185. https://doi.org/10.1007/s00707-017-1957-6

[27] Merryisha, Samuel, and Parvathy Rajendran. "Experimental and CFD Analysis of Surface Modifiers on Aircraft Wing: A Review." CFD Letters 11, no. 10 (2019): 46-56. 